\title{
An update on the use of inhaled therapy in COPD
}

\author{
Authors: Oliver Price, ${ }^{A}$ Chandra Sarkar ${ }^{B}$ and Shruthi Konda ${ }^{C}$
}

Inhaled corticosteroids represent the mainstay of pharmacological treatment for reversible airways disease, and traditionally have had widespread use in non-reversible chronic obstructive pulmonary disease (COPD). However, use in this group may expose patients to an increased risk of pneumonia, without significant benefit to symptoms or exacerbation frequency. Here we provide a guide to the use of inhaled therapy in COPD for the general physician.

KEYWORDS: COPD, inhaled corticosteroids

\section{Introduction}

Chronic obstructive pulmonary disease (COPD) is an airways disorder, characterised by airflow obstruction (forced expiratory volume in 1 second $\left[\mathrm{FEV}_{1}\right]$ / forced vital capacity $[\mathrm{FVC}]<0.7$ ) on spirometry, which lacks significant reversibility on appropriate testing. It affects an estimated 3 million people in the UK, with approximately 900,000 having received a formal diagnosis. ${ }^{1}$ Symptoms typically consist of cough, dyspnoea and wheeze, which do not vary significantly over time, in contrast to asthma, where symptoms may differ over hours to days.

The predominant aetiological agent is cigarette smoking, with increasing recognition of alternative exposures, for example biomass fuel, as causative, even in never smokers.

Diagnosis requires a combination of suggestive history, examination, imaging and spirometry, where findings of $\mathrm{FEV}_{1} / \mathrm{FVC}$ $<0.7, \mathrm{FEV}_{1}<80 \%$ predicted, are supportive.

Therapy is multifaceted including inhaled pharmacotherapy which is discussed below. Particular importance is also placed on smoking cessation and pulmonary rehabilitation programmes to halt further damage to lung tissue and maintain exercise performance. Flu vaccination is recommended to reduce infection risk.

The principle agents of inhaled therapy include short-acting beta-agonists (SABA, eg salbutamol), long-acting beta-agonists (LABA, eg formoterol), long-acting muscarinic antagonist (LAMA eg tiotropium) and inhaled corticosteroids (ICSs, eg fluticasone). Numerous combination devices containing two or three agents exist.

Authors: ${ }^{A}$ clinical fellow, respiratory medicine, Watford General Hospital, Watford, UK; ${ }^{\text {B }}$ general practitioner, City \& Hackney GP Confederation, London, UK; ${ }^{C}$ consultant physician, respiratory medicine, Watford General Hospital, Watford, UK

\section{Inhaled therapy: the historic approach and its problems}

As recently as 2010, National Institute for Health and Care Excellence (NICE) guidelines advocated a spirometry-based approach to inhaled therapies where, in the presence of appropriate symptoms, ICS in combination with LABA was commenced in those with an $\mathrm{FEV}_{1}<50 \%$, with or without LAMA. This has led to a number of patients on 'triple therapy' of LABA/ ICS plus LAMA.

Adverse effects of ICS such as dysphonia and oral candida infection are long established; however, more recently, a link between ICS and pneumonia has emerged. A comprehensive Cochrane review of 43 studies including 30,000 patients, using either fluticasone or budesonide demonstrated an increase in serious (requiring hospital admission) episodes of pneumonia (fluticasone, odds ratio [OR] 1.78, 95\% confidence interval [CI] 1.50-2.12, budesonide OR 1.62, 95\% CI 1.00-2.62) relative to those not on an ICS. ${ }^{2} \mathrm{~A}$ higher total number of pneumonia episodes (hospital or community treated) were seen with fluticasone use compared to budesonide (OR 1.86, 95\% CI 1.04-3.34). However, there was no increase in mortality seen in ICS users versus not, as deaths were so infrequent.

\section{Inhaled therapy: the current approach}

More recently, the international collaboration Global Initiative for Chronic Obstructive Lung Disease (GOLD) ${ }^{3}$ has updated its treatment recommendations to reflect this. A symptom-based approach to severity stratification is recommended in favour of the previously used $\mathrm{FEV}_{1}$, acknowledging the weak correlation between $\mathrm{FEV}_{1}$, symptoms and impairment of health, and allowing for more individualised patient care.

Once a diagnosis of COPD is confirmed, patients are stratified according to Fig 1.

Patients are placed into an ABCD category based on level of dyspnoea according to modified medical research council scale (mMRC, $0-1$ or $\geq 2$,) or COPD assessment test, (CAT, $<10, \geq 10$ ) and frequency and severity of exacerbations, (0-1 per year with no hospital admission, or either $\geq 1$ with hospital admission or $\geq 2$ ).

Inhaled therapy is prescribed according to the severity score, as per Fig 2.

$>$ Group A patients (low dyspnoea, low exacerbation frequency) are treated with a bronchodilator of the prescribers choosing.

> Group B (low exacerbation frequency, high dyspnoea) are offered either LABA or LAMA as first line, with a combination LABA/LAMA should dyspnoea persist. 
Fig 1. Symptom stratification. Adapted with permission from $\mathrm{GOLD}^{3}{ }^{3} \mathrm{FEV}_{1}=$ forced expiratory volume in the first second; $\mathrm{FVC}=$ forced vital capacity; $\mathrm{mMRC}=$ modified Medical Research Council; CAT = COPD assessment test

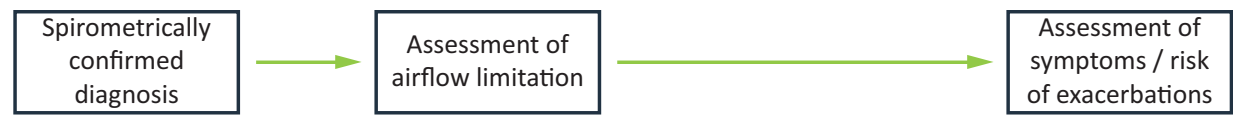

\begin{tabular}{|c|c|c|}
\cline { 2 - 3 } \multicolumn{1}{c|}{} & \multicolumn{2}{|c|}{ FEV $_{1}$ (\% of predicted) } \\
\hline $\begin{array}{c}\text { Post-bronchodilator } \\
\text { FEV }_{1} / \text { FVC }<0.7\end{array}$ & GOLD 1 & $\geq 80$ \\
\hline GOLD 2 & $50-79$ \\
\hline GOLD 3 & $30-49$ \\
\hline GOLD 4 & $<30$ \\
\hline
\end{tabular}

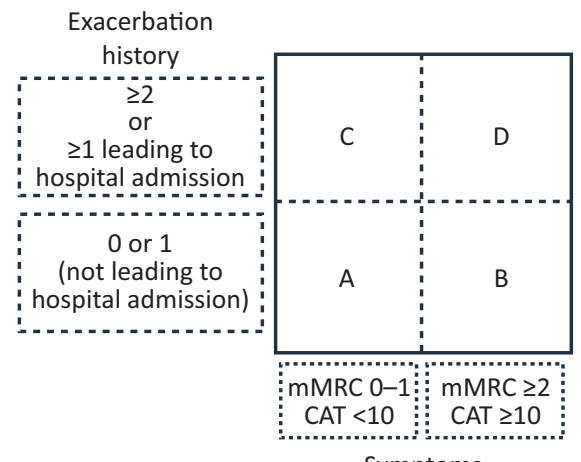

Symptoms
> Group C (high exacerbation frequency, low dyspnoea) are offered LAMA as first line, based on evidence suggesting reduced exacerbation frequency with LAMA in preference to LABA, ${ }^{4}$ with LABA/LAMA as second line.

> Finally, Group D (high exacerbation frequency, high dyspnoea) are offered LABA/LAMA as first line, with LABA/LAMA

ICS reserved only for those who still experience frequent exacerbations despite LABA/LAMA.

\section{Inhaled therapy: a new approach - ICS vs LABA/LAMA}

There is an increasing body of evidence supporting the use of LABA/LAMA in place of ICS/LABA, while maintaining benefits across a range of outcomes, including lung function, health status and exacerbation rates.

Meta-analysis by Rodrigo et al reviewing various outcomes in those treated with ICS/LABA vs LABA/LAMA (and LAMA alone) pooled data from 23 RCTs, which included over 20,000 patients, including all currently available LABA/LAMA combinations, focusing specifically on doses and combinations available for prescription. $^{5}$

This demonstrated numerous benefits when using LABA/LAMA, relative to LABA/ICS. An $80 \mathrm{~mL}$ improvement at week 12 and week 24-26 trough $\mathrm{FEV}_{1}$ was noted, although this was below the minimum clinically important difference (MCID) of $100 \mathrm{~mL}$. A $44 \%$ increase in likelihood of achieving MCID was seen. Peak FEV was also higher at 12 weeks, with a mean difference of $120 \mathrm{~mL}$. However, this does not inform on changes to lung function over the longer term.

Rescue medication use was reduced at the end of the study period (12-26 weeks) by -0.18 puffs/day, ( $95 \%$ CI -0.28 to -0.07 , $p=0.001$, ) in the LABA/LAMA group.

LABA/LAMA demonstrated a significant reduction in annual rates of both moderate and severe exacerbations (relative risk [RR] $0.82,95 \%$ CI $0.75-0.91, p<0.001$.) Although both the two included studies used indacaterol/glycopyrronium, hence it was not possible to determine whether this was a class effect or specific to this combination.
Fig 2. Inhaled therapy. Adapted with permission from $\mathrm{GOLD}^{3} \mathrm{FEV}_{1}=$ forced expiratory volume in the first second; ICS = inhaled corticosteroids; $\mathrm{LABA}=$ long-acting beta-agonist; LAMA = long-acting muscarinic antagonist
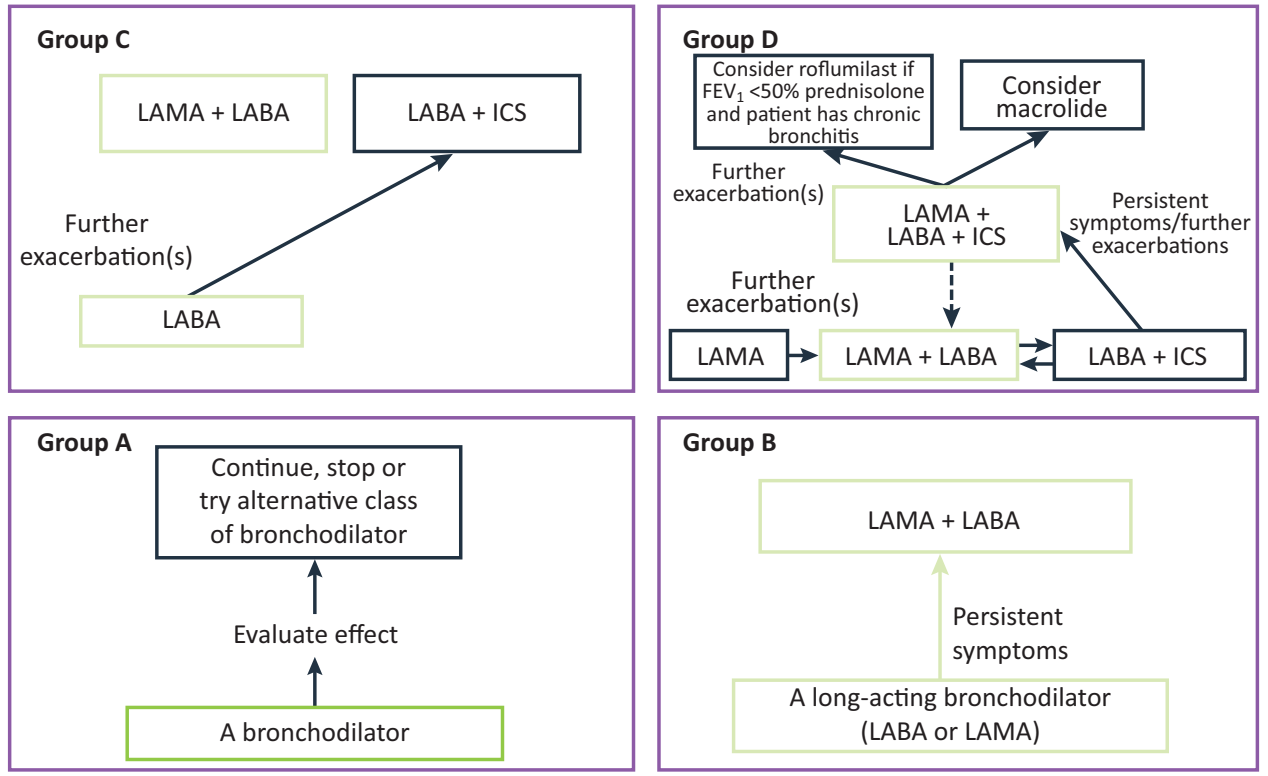
Fewer adverse events (RR 0.94, 95\% CI 0.89-0.99, $\mathrm{p}=0.02$ ) and fewer incidences of pneumonia (RR $0.59,95 \%$ CI $0.43-0.81$. $\mathrm{p}=0.001$ ) were seen in the LABA/LAMA group. No significant difference was observed in the rates of serious adverse events or death.

Some limitations are noted to this meta-analysis. Included studies tended to enrol stable patients, with infrequent exacerbations, although there were exceptions including the two studies with exacerbation frequency as the primary outcome. Study periods tended to be no longer than $52 / 64$ weeks so conclusions into mortality and disease progression could not be drawn. The LABA/ICS used was limited to just salmeterol / fluticasone propionate. Fluticasone propionate has been linked to an increased risk of pneumonia relative to budesonide, ${ }^{2}$ and so this may have affected results. Finally, there were no head-to-head studies of the different LABA/LAMA combinations, so individual efficacy cannot be established.

Published more recently than the above meta-analysis, the IMPACT study, ${ }^{6}$ which included 10,355 patients randomised to either LABA/LAMA/ICS, LABA/ICS or LABA/LAMA, each in a single inhaler device. The primary endpoint was annual rate of moderate to severe exacerbations, with a rate of 0.91 in the triple-therapy arm, compared to 1.21 in the LABA/LAMA group. Trough FEV was $54 \mathrm{~mL}(95 \%$ CI 85-109, $p<0.001)$ greater in the triple-therapy group. St George's Respiratory Questionnaire (SGRQ) score was -1.8 points ( $95 \% \mathrm{CI}-2.6--1.0 p<0.001$ ). Both of these outcome measures were below the MCID. Fewer episodes of pneumonia were noted in the LABA/LAMA group relative to triple therapy, with a time to first incidence hazard ratio of 1.53 (95\% CI 1.22-1.92, $\mathrm{p}<0.001)$. While this large randomised study does demonstrate a significant reduction in exacerbation frequency on a population level, the rate reduction is 0.3 events per year, (0.91 vs 1.21$)$ and so may not be of such importance on an individual patient level.

\section{Inhaled therapy: a new approach - ICS withdrawal}

An older meta-analysis of early trials looking at ICS withdrawal in those already established on it, noted methodological limitations such as lack of bias and quality assessment, and inconsistent definition and management of exacerbations, although reported a 1.11 (95\% CI 0.84-1.46) times increase in likelihood of exacerbation in the following year in the ICS withdrawal group, and overall concluded no evidence for clinically important deterioration following ICS withdrawal. ${ }^{7}$

A more recent meta-analysis concluded no significant increase in exacerbation rates post withdrawal, athough a clinically important increase in risk of severe exacerbation was detected. ${ }^{8}$ Decline in $\mathrm{FEV}_{1}(-30 \mathrm{~mL})$ and SGRQ (+1.24 points) was noted, although these were both below the MCID.

Included in the above meta-analysis was the 12-month doubleblind WISDOM study. ${ }^{9}$ Patients with severe or very severe COPD, who had experienced at least one exacerbation in the previous year were included. It compared continuation of triple therapy following a 6 -week run-in period, versus a stepwise reduction to eventual discontinuation of ICS, and demonstrated non-inferiority (HR 1.06; 95\% CI 0.94-1.19 - pre-specified non-inferiority criterion of 95\% CI 1.20) with regards to first moderate or severe exacerbation. Note was made of a reduction in $\mathrm{FEV}_{1}$ in the ICS withdrawal group at 18 weeks (when ICS withdrawal was complete) and at the end of the 52-week study period, although this was not felt to be clinically significant as it was below the MCID.
Contradicting these findings, however, the shorter 26-week INSTEAD trial, which compared switching from ICS/LABA to LABA alone, in moderate COPD patients who had experienced no exacerbations in the previous year demonstrated just $9 \mathrm{~mL}$ (95\% CI - 45-26 mL) difference between the two groups. ${ }^{10}$ There was no statistical difference in exacerbation rates.

In the CRYSTAL study, patients were randomised to direct switch to LABA/LAMA, or continuation of current therapy. ${ }^{11}$ Compared to the LABA/ICS group, a $71 \mathrm{~mL}$ improvement in trough $\mathrm{FEV}_{1}$ was noted, with significantly more patients reaching the MCID However, this was an open-label study, with a duration of just 12 weeks, so conclusions regarding the impact on lung function over the longer term could not be drawn.

\section{Eosinophilia}

Accepting the limitations of these studies, the above demonstrates that ICS withdrawal is safe in terms of effects on exacerbation rates and lung function; however, one caveat to withdrawal relates to those patients with an eosinophilic phenotype. Post-hoc analysis ${ }^{12}$ of data from the aforementioned WISDOM study demonstrated that exacerbation rates in the ICS withdrawal group was higher for those with higher peripheral eosinophil counts, $2 \%$ or greater (RR 1.22 [95\% CI 1.02-1.48]), $4 \%$ or greater (1.63 [1.19-2.24]), and 5\% or greater (1.82 [1.20-2.76]), with a cut-off of $4 \%$ / 300 cells per $\mu \mathrm{L}$, thought to predict a poor response to ICS withdrawal.

Post-hoc analysis ${ }^{13}$ of two randomised double-blind placebo controlled trials in which ICS/LABA was compared to LABA alone also demonstrated an increased exacerbation frequency with increased peripheral eosinophil count -0.89 for $<2 \%, 1.21(2-4 \%)$, $1.24(4-6 \%), 1.62$ (>6\%). ICS use was associated with a $29 \%$ decrease in annual exacerbation rate in those with eosinophils $>2 \%$, but a $10 \%$ decrease in those with eosinophils $<2 \%$.

Post-hoc analysis of the 48-week randomised double-blind FORWARD study divided patients into quartiles based on baseline eosinophil count. ${ }^{14}$ While exacerbation rates among patients receiving ICS were similar across the quartiles, within the highest quartile $(\geq 279.8 / \mu \mathrm{l})$ a $46 \%$ reduction in exacerbation rate was seen in those using ICS.

A prospective population-based study in Copenhagen examined the relationship between baseline eosinophil count and COPD exacerbations and demonstrated that eosinophil levels above $0.34 \times 10^{9}$ cells $/ L$ were associated with a 1.76 -fold increased risk of severe exacerbations (95\% CI 1.56-1.99). ${ }^{15}$

However, contrary to the above, post-hoc analysis of FLAME data showed that LABA/LAMA was superior to ICS/LAMA for the prevention of exacerbations, with no relationship demonstrated between exacerbation frequency and eosinophil count. ${ }^{16}$ Higher rates of pneumonia were noted in the ICS/LABA group regardless of eosinophils.

The IMPACT study demonstrated fewer exacerbations in those on triple therapy versus $L A B A / L A M A$, although interestingly also versus those on ICS/LABA, regardless of eosinophil count. ${ }^{6}$ A greater reduction in exacerbation rate was noted, however, in those with an eosinophil count greater than 150 cells/ $\mu \mathrm{L}$.

There is no clear consensus regarding cut-off values for eosinophils to be used to predict COPD exacerbations, or indeed whether eosinophil counts can be used in this way at all. It may, however, be reasonable to consider patients with an eosinophilia, together with a history of recurrent exacerbations, to be at increased risk of future exacerbations, and so continue ICS in this patient group. 


\section{Method of withdrawal}

With regards to method of withdrawal - sudden stop versus gradual reduction, while the WISDOM study used a gradual reduction in ICS dose, additional studies have adopted a sudden discontinuation approach. There is no strong evidence to support gradual reduction, ${ }^{17}$ and a direct switch from LABA/ICS to LABA/ LAMA can be made.

\section{Conclusion}

We have shown that ICS use is associated with an increased risk of pneumonia, especially when using fluticasone, and that LABA/ LAMA as an alternative is safe and effective across a range of outcome measures.

ICS use in the management of COPD should now be regarded not as first line, but more of an 'if all else fails' treatment, in those who continue to exacerbate despite using LABA/LAMA.

A stepwise approach could be considered: as LAMA as first line, followed by combination LABA/LAMA should dyspnoea or exacerbation frequency not be satisfactorily controlled, and finally LABA/LAMA/ICS reserved for only a select group of patients troubled by recurrent exacerbations despite dual bronchodilators.

In those already established on LABA/ICS, direct withdrawal of ICS and switch to LABA/LAMA can be undertaken. Care should be taken when considering ICS withdrawal in patients who suffer frequent exacerbations, especially those who have a raised peripheral eosinophil count, although cut-off levels are not clear, and the relationship between eosinophilia and exacerbation risk remains to be elucidated.

\section{References}

1 National Institute for Health and Care Excellence. Chronic obstructive pulmonary disease in over 16s: diagnosis and management [CG101]. London: NICE, 2010.

2 Kew KM, Seniukovich A. Inhaled steroids and risk of pneumonia for chronic obstructive pulmonary disease. Cochrane Database Syst Rev 2014;3:CD010115.

3 From the Global Strategy for the Diagnosis, Management and Prevention of COPD, Global Initiative for Chronic Obstructive Lung Disease (GOLD) 2017. http://goldcopd.org.

4 Vogelmeier C, Hederer B, Glaab T et al. Tiotropium versus salmeterol for the prevention of exacerbations of COPD. N Engl J Med 2011;364:1093-103.

5 Rodrigo G], Price D, Anzueto A et al. LABA/LAMA combinations versus LAMA monotherapy or LABA/ICS in COPD: a systematic review and meta-analysis. Int J Chron Obstruct Pulmon Dis 2017;12:907-22.
6 Lipson DA, Barnhart F, Brealey $\mathrm{N}$ et al. Once-daily single-inhaler triple versus dual therapy in patients with COPD. N Engl J Med 2018:378:1671-80.

7 Nadeem NJ, Taylor S], Eldridge SM. Withdrawal of inhaled corticosteroids in individuals with COPD - a systematic review and comment on trial methodology. Respir Res 2011;12:107.

8 Calzetta L, Matera MG, Braido F et al. Withdrawal of inhaled corticosteroids in COPD: a meta-analysis. Pulm Pharmacol Ther 2017:45:148-58.

9 Magnussen H, Disse B, Rodriguez-Roisin R et al. Withdrawal of inhaled glucocorticoids and exacerbations of COPD. N Engl ] Med 2014;371:1285-94.

10 Rossi A, van der Molen T, del Olmo R et al. INSTEAD: a randomised switch trial of indacaterol versus salmeterol/fluticasone in moderate COPD. Eur Respir ] 2014;44:1548-56.

11 Vogelmeier CF, Gaga M, Aalamian-Mattheis M et al. Efficacy and safety of direct switch to indacaterol/glycopyrronium in patients with moderate COPD: the CRYSTAL open-label randomised trial. Respir Res 2017;18:140.

12 Watz $\mathrm{H}$, Tetzlaff $\mathrm{K}$, Wouters EF et al. Blood eosinophil count and exacerbations in severe chronic obstructive pulmonary disease after withdrawal of inhaled corticosteroids: a post-hoc analysis of the WISDOM trial. Lancet Respir Med 2016;4:390-8.

13 Pascoe S, Locantore N, Dransfield M, Barnes N, Pavord I. Blood eosinophil counts, exacerbations, and response to the addition of inhaled fluticasone furoate to vilanterol in patients with chronic obstructive pulmonary disease: a secondary analysis of data from two parallel randomised controlled trials. Lancet Respir Med 2015;3:435-42.

14 Siddiqui SH, Guasconi A, Vestbo ] et al. Blood Eosinophils: a biomarker of response to extrafine beclomethasone/formoterol in chronic obstructive pulmonary disease. Am J Respir Crit Care Med 2015;192:523-25.

15 Vedel-Krogh S, Nielsen SF, Lange P, Vestbo J, Nordestgaard BG. Blood eosinophils and exacerbations in chronic obstructive pulmonary disease. The Copenhagen General Population Study. Am J Respir Crit Care Med 2016;193:965-74.

16 Roche N, Chapman KR, Vogelmeier CF et al. Blood eosinophils and response to maintenance chronic obstructive pulmonary disease treatment. Data from the FLAME trial. Am J Respir Crit Care Med 2017;195:1189-97.

17 Miravitlles M, Cosío BG, Arnedillo A et al. A proposal for the withdrawal of inhaled corticosteroids in the clinical practice of chronic obstructive pulmonary disease. Respir Res 2017;18:198.

Address for correspondence: Dr Oliver Price, Watford General Hospital, Vicarage Road, Watford WD18 0HB, UK. Email: oliver.price@nhs.net 